\title{
Bioinformatics identification of microRNAs involved in polycystic ovary syndrome based on microarray data
}

\author{
YAN HOU ${ }^{1}$, YAOQIN WANG ${ }^{2}$, SUMING XU $^{2}$, GAIMEI QI $^{2}$ and XUEQING WU ${ }^{1,2}$ \\ ${ }^{1}$ The Second Hospital of Shanxi Medical University Shanxi Medical University, Taiyuan, \\ Shanxi 030001; ${ }^{2}$ Center of Reproductive Medicine, Children's Hospital of Shanxi and \\ Women Health Center of Shanxi, Taiyuan, Shanxi 030013, P.R. China
}

Received October 10, 2018; Accepted April 9, 2019

DOI: $10.3892 / \mathrm{mmr} .2019 .10253$

\begin{abstract}
Polycystic ovary syndrome (PCOS) is the most common endocrine disease in women of reproductive age. MicroRNAs (miRNAs or miRs) serve important roles in the physiological and pathological process of PCOS. To identify PCOS-associated miRNAs, the dataset GSE84376 was extracted from the Gene Expression Omnibus database. Differentially expressed miRNAs (DE-miRNAs) were obtained from Gene-Cloud Biotechnology Information and potential target genes were predicted using TargetScan, DIANA-microT-CDS, miRDB and miRTarBase tools. Gene Ontology enrichment analysis was performed using Metascape and a protein-protein interaction network was constructed using Cytoscape. Transcription factors were obtained from FunRich. DE-miRNAs were verified by reverse transcription-quantitative PCR. At the screening phase, there were seven DE-miRNAs in the PCOS group not present in the control group. In total, 935 target genes were identified, which are involved in the development and maturation of oocytes. Mitogen-activated protein kinase 1, phosphatase and tensin homolog, cAMP responsive element binding protein 1, signal transducer and activator of transcription 3, interferon $\gamma$, Fms-related tyrosine kinase 1, transcription factor p65, insulin receptor substrate 1 , DnaJ homolog superfamily $\mathrm{C}$ member 10 and casein kinase $2 \alpha 1$ were identified as the top 10 hub genes in the protein-protein interaction network. Specificity protein 1 was the most enriched transcription factor. At the validation phase, the levels of Homo sapiens (hsa)-miR-3188 and hsa-miR-3135b were significantly higher in the PCOS group than in the control group. In addition, the expression level of hsa-miR-3135b was significantly correlated with the number of oocytes retrieved, the fertilization rate and the
\end{abstract}

Correspondence to: Dr Xueqing Wu, Center of Reproductive Medicine, Children's Hospital of Shanxi and Women Health Center of Shanxi, 13 Xinmin North Street, Taiyuan, Shanxi 030013, P.R. China E-mail: xueqingwu416@126.com

Key words: microRNAs, polycystic ovary syndrome, bioinformatic analysis cleavage rate $(\mathrm{P}<0.05)$. The present bioinformatics study on miRNAs may offer a novel understanding of the mechanism of PCOS, and may serve to identify novel miRNA therapeutic targets.

\section{Introduction}

Polycystic ovary syndrome (PCOS) is a common endocrine disorder with an incidence of 6-21\% $(1,2)$. PCOS affects women of reproductive age and symptoms include light menstruation or even amenorrhea, hyperandrogenemia and polycystic ovarian morphology in addition to metabolic disorders, including insulin resistance, diabetes and obesity (3). PCOS is a primary cause of female infertility due to a failure in oocyte-follicle maturation (4).

Granulosa cells (GCs) serve an important role in oocyte development and maintenance of the oocyte microenvironment by secreting steroid hormones and producing growth hormones. Additionally, GCs are the primary sites of estrogen synthesis and provide endocrine signaling to other tissues (5). A previous study demonstrated that dysfunction of GCs contributes to abnormal folliculogenesis in patients with PCOS (6). GCs provide nutrients and growth regulators to the oocyte. Therefore, it is important to investigate the role of GCs in the development of PCOS.

Previous studies have indicated that numerous genes participate in the pathogenesis of PCOS (7-11). A previous study revealed that co-activators of estrogen receptors, including nuclear receptor co-activator (NCOA) 1, NCOA2, NCOA3, CREB binding protein, histone acetyltransferase p300, lysince acetyltransferase $2 \mathrm{~B}$ and coactivator associated arginine methyltransferase 1, as well as co-repressors such as nuclear receptor corepressor 1 and nuclear receptor corepressor 2, were deregulated in cumulus cells from patients with PCOS, which may contribute to the etiology of PCOS (7). Jansen et al (8) demonstrated that androgen serves an important role in the pathogenesis of PCOS through a microarray analysis of PCOS compared with normal ovaries. In subcutaneous adipose tissue from PCOS, C-C motif ligand 2 and heme oxygenase 1 were expressed at high levels, while adiponectin receptor 2 , lipoprotein lipase and twist-related protein 1 were expressed at low levels. These molecules were associated with lipid metabolism, insulin sensitivity, inflammation and oxidative stress (9). 
However, how these genes are post-transcriptionally regulated is poorly understood.

An improved understanding regarding the regulation of genes has resulted from the identification of microRNAs (miRNAs or miRs). These small, non-coding RNAs of 20-22 nucleotides in length are capable of inhibiting protein translation or degrading target mRNAs in multiple biological and pathological processes, including in cell proliferation, apoptosis, differentiation, cell cycle control and metabolism $(12,13)$. Kim et al (14) demonstrated that miR-27a, miR-let-7c and miR-322 can regulate the meiotic competence of oocytes during the in vitro maturation of mouse follicles. A previous study indicated that different miRNAs are expressed in cumulus cells in patients with PCOS compared with healthy controls (15). A number of previous studies have also reported that miRNAs serve important roles in ovarian function and GC apoptosis (16-18). Abnormal expression of miRNAs is also associated with several reproductive disorders (19-22). Murri et al (23) identified that circulating miRNA-21, miRNA-27b, miRNA-103 and miRNA-155 are differentially expressed between patients with PCOS and healthy controls, and are involved in the pathogenesis of PCOS, including inflammatory processes, hormone metabolism and insulin signaling. Furthermore, Shi et al (24) indicated that miR-483-5p is important to reduce insulin resistance, while miR-486-5p may promote cumulus cell proliferation by activating PI3K/AKT signaling in patients with PCOS. Jiang et al (25) reported that miRNA-93 could promote ovarian GC proliferation by targeting cyclin-dependent kinase inhibitor $1 \mathrm{~A}$ in patients with PCOS. Zhu et al (26) indicated that miRNA-34a promoted ovarian GC apoptosis by inhibiting $\mathrm{B}$ cell lymphoma-2 (Bcl-2) and increasing the expression of $\mathrm{Bcl}-2$-associated $\mathrm{X}$ protein and caspase- 3 . However, to date, little is known about the miRNA profiles in GCs in patients with PCOS.

The present study screened for differentially expressed miRNAs (DE-miRNAs) from the dataset GSE84376. Target genes of the DE-miRNAs were predicted and their potential functions were analyzed by Gene Ontology (GO) enrichment analysis. Furthermore, a protein-protein interaction (PPI) network was constructed to identify hub genes. Finally, screening was conducted to identify transcription factors that may regulate the target genes. The present bioinformatics study on miRNAs may provide a novel perspective into the pathological mechanism of PCOS.

\section{Materials and methods}

miRNA microarray analysis. The present study used 'miRNA, granulosa cells, PCOS, human' as key words to extract expression data for GCs from patients with PCOS and controls in the National Center for Biotechnology Information Gene Expression Omnibus (GEO) database (https://www.ncbi. nlm.nih.gov/geo/query/acc.cgi?acc=GSE84376; accessed on December 2017). The dataset GSE84376, based on Affymetrix Multispecies miRNA-3 Array platform (Affymetrix; Thermo Fisher Scientific, Inc.) contained 15 cases of PCOS and 13 cases of non-PCOS. The raw data were uploaded to the website of Gene-Cloud of Biotechnology Information (GCBI; https://www.gcbi.com.cn/gclab/html/index; Genminix Informatics Co., Ltd.) for analysis. GCBI, a good online bioinformatics analysis platform, combines a variety of sample information, research findings, genetic information, bioinformatics and data algorithms to create a 'gene knowledge base'. The GCBI platform can be used to systematically analyze GEO dataset-derived gene expression information. Cluster analysis was performed on DE-miRNAs using a fold-change $>2$ and false discovery rate $\leq 0.05$ as cut off values.

Prediction of target genes. Data mining tools, including three different target prediction databases [TargetScan (http://www.targetscan.org/vert_71/), DIANA-microT-CDS (http://diana.imis.athena-innovation.gr/DianaTools/index.php? $\mathrm{r}=$ site/page $\& \mathrm{view}=$ software) and $\mathrm{miRDB}$ (http://mirdb. org/miRDB/)] and a database containing validated targets (miRTarBase; http://mirtarbase.mbc.nctu.edu.tw/php/index. php) were used to predict target genes of DE-miRNAs. According to the parameters set for each bioinformatics tool, the predicted genes were selected by $\geq 3$ algorithms. Then, target genes were illustrated from each tool using Venny 2.1 (http://bioinfogp.cnb.csic.es/tools/venny/index.html).

Gene ontology $(G O)$ enrichment analysis. GO was used for gene functional enrichment analysis using Metascape software (http://metascape.org). The resulting GO terms with $\mathrm{P}<0.05$ were considered significantly enriched in the differentially expressed genes.

PPI network. To further investigate the pathogenesis of PCOS, a PPI network was constructed using Cytoscape software (version 3.6.1) (27). An integrated score $>0.4$ [the default threshold in the STRING database; http://www.string-db. org/ (28)] was defined to construct the PPI network. Then, the topological structure of the network was analyzed and the degree for each gene was calculated. Hub genes were defined as those with degree $\geq 10$.

Screening of potential transcription factors. The study identified the enriched transcription factors regulating target genes of DE-miRNA using FunRich software (version 3.1.3; http://www.funrich.org) (29), a functional enrichment and interaction network analysis tool.

Enrollment of subjects. All the patients enrolled in the present study underwent in vitro fertilization (IVF)/intracytoplasmic sperm injection-embryo transfer at the Center of Reproductive Medicine, Children's Hospital of Shanxi and Women Health Center of Shanxi between January 2018 to July 2018. According to the 2003 Rotterdam Revised Criteria for PCOS (30), 38 patients with PCOS and 35 patient controls with male or tubal infertility factors were enrolled into the present study. Patients with prolactinoma, congenital adrenal hyperplasia, thyroid disorder, Cushing syndrome and adrenal tumors were excluded. The subjects did not receive any hormonal treatments 3 months prior to enrollment. The present study was approved by the Ethics Committee of Children's Hospital of Shanxi and Women Health Center of Shanxi. Written informed consent for inclusion was obtained from each participant.

The demographic and clinical data, including age, infertility duration, height, weight and serum levels of sex hormones [luteinizing hormone ( $\mathrm{LH})$, follicle-stimulating hormone (FSH), estrogen (E2), progesterone (P) and testosterone (T)] 
Table I. Comparison of clinical characteristics between the PCOS and control groups.

\begin{tabular}{lcrr}
\hline Variables & PCOS group $(\mathrm{n}=38)$ & Control group $(\mathrm{n}=35)$ & P-value \\
\hline Age (year) & $29.60 \pm 0.66$ & $29.66 \pm 0.82$ & 0.960 \\
Infertility duration (year) & $4.62 \pm 0.50$ & $4.64 \pm 0.55$ & 0.974 \\
Menarche age (year) & $13.29 \pm 0.17$ & $13.46 \pm 0.18$ & 0.493 \\
Body mass index (Kg/m $\left.{ }^{2}\right)$ & $25.25 \pm 0.59$ & $22.63 \pm 0.53$ & 0.002 \\
Basal hormone levels & & & \\
LH (mIU/ml) & $8.10 \pm 0.69$ & $5.50 \pm 0.59$ & 0.005 \\
FSH (mIU/ml) & $6.94 \pm 0.32$ & $8.71 \pm 0.61$ & 0.013 \\
E2 (pg/ml) & $90.43 \pm 11.85$ & $88.80 \pm 12.95$ & 0.926 \\
P (ng/ml) & $0.62 \pm 0.09$ & $0.50 \pm 0.06$ & 0.275 \\
T (ng/ml) & $45.09 \pm 3.66$ & $41.58 \pm 3.60$ & 0.498 \\
LH/FSH & $1.21 \pm 0.10$ & $0.66 \pm 0.07$ & $<0.001$ \\
IVF outcomes & & & 0.003 \\
No. of oocytes retrieved & $25.53 \pm 2.22$ & $17.09 \pm 1.58$ & 0.093 \\
MII oocyte rate & $0.75 \pm 0.03$ & $0.82 \pm 0.03$ & $0.01 \pm 0.02$ \\
Fertilization rate & $0.72 \pm 0.03$ & $0.92 \pm 0.02$ & $0.57 \pm 0.04$ \\
Cleavage rate & $0.85 \pm 0.03$ & $0.57 \pm 0.03$ & 0.049 \\
Embryo rate & & & 0.909 \\
\hline
\end{tabular}

Data are presented as the mean \pm SEM. $\mathrm{P}<0.05$ was considered to indicate a statistically significant difference. PCOS, polycystic ovary syndrome; LH, luteinizing hormone; FSH, follicle stimulating hormone; E2, estrogen; P, progesterone; T, testosterone; MII, metaphase II; IVF, in vitro fertilization.

were recorded (Table I). Reproductive laboratory data were also obtained, including the number of retrieved oocytes, metaphase II (MII) oocytes, fertilized oocytes, cleavage rate and embryos.

Isolation of GCs from follicular fluid. GCs were collected from the follicular fluid during oocyte collection 34-36 h after human chorionic gonadotrophin (hCG) injection. The cumulus-oocyte complexes were used for IVF. The remaining follicular fluid was transferred to $4^{\circ} \mathrm{C}$ within $1 \mathrm{~h}$. The follicular fluid was prepared by centrifugation at $400 \mathrm{x}$ g for $5 \mathrm{~min}$ followed by layering on $40 \%$ Percoll $^{\circledR}$ (Sigma-Aldrich; Merck $\mathrm{KGaA}$ ) at room temperature. Following this, three layers could be distinguished; the middle ring-like layer was collected and washed by centrifugation at $400 \mathrm{x}$ g for $10 \mathrm{~min}$. The pellet was resuspended with $1 \mathrm{X}$ PBS and red blood cell lysate was added to a volumetric ratio of $1: 3$ and kept at room temperature for $10 \mathrm{~min}$. Following centrifugation at 2,860 $\mathrm{x} \mathrm{g}$ for $1 \mathrm{~min}$ at room temperature, three wash steps were carried out using PBS and centrifugation at 2,860 $\mathrm{x}$ g for $1 \mathrm{~min}$ at room temperature, and GCs were collected.

RNA extraction and reverse transcription-quantitative $(R T-q) P C R$. Total RNA was extracted from the purified human GCs using an miRNeasy Mini kit (Qiagen $\mathrm{GmbH}$ ) according to the manufacturer's protocol. cDNA synthesis was performed using the miScript II RT kit (Qiagen $\mathrm{GmbH}$ ) following the manufacturer's temperature protocol: $37^{\circ} \mathrm{C}$ for $60 \mathrm{~min}$ and $95^{\circ} \mathrm{C}$ for $5 \mathrm{~min}$. PCR, using miR-specific primers and universal adaptor PCR primers (iGeneBio Co; GeneCopoeia, Inc.), was performed using a miScript SYBR
Green PCR kit (Qiagen $\mathrm{GmbH}$ ) according to the manufacturer's protocol using a CFX Connect Real-time System (Bio-Rad Laboratories, Inc.). The miR-specific primers were as follows: Hsa-miR-3135b AGCGAGTGCAGTGGTGAAA; hsa-miR-4433-3p GAGTGGGGGTGGGACATA AA; hsa-miR-3188 GTGCGGATACGGGGAAAA; hsa-miR-1587 TGGGCTGGGTTGGGAAA; hsa-miR-1225-5p GGCCCA GTGGGGGGAA; hsa-miR-4749-5p ACAGGCCAGGGC ATCAAA; hsa-miR-4417 GGCTTCCCGGAGGGAAA. The PCR conditions were as follows: $95^{\circ} \mathrm{C}$ pre-denaturation for $15 \mathrm{~min}$, followed by 40 cycles of denaturation at $94^{\circ} \mathrm{C}$ for $15 \mathrm{sec}$, annealing at $55^{\circ} \mathrm{C}$ for $30 \mathrm{sec}$ and extension at $70^{\circ} \mathrm{C}$ for $30 \mathrm{sec}$. Relative gene expression was analyzed using the $2^{-\Delta \Delta \mathrm{Cq}}$ method (31). U6 small nuclear RNA was used as a miRNA internal control. miRNA and U6 (cat. no. HmiRQP9001) primers were purchased from iGeneBio (GeneCopoeia, Inc.).

Statistical analysis. Statistical analyses were performed using SPSS version 17.0 (SPSS, Inc.). Differences between groups were assessed by Student's t-test (2-sided). Pearson's correlation coefficient was used to assess correlations between variables. All quantitative results from at least three independent experiments are presented as the mean \pm SEM. A two-tailed $\mathrm{P}<0.05$ was considered to indicate a statistically significant difference. All statistical graphs were generated using GraphPad Prism 6.0 (GraphPad Software, Inc.).

\section{Results}

Identification of DE-miRNAs and their target genes. There were seven upregulated DE-miRNAs in GCs of patients with 


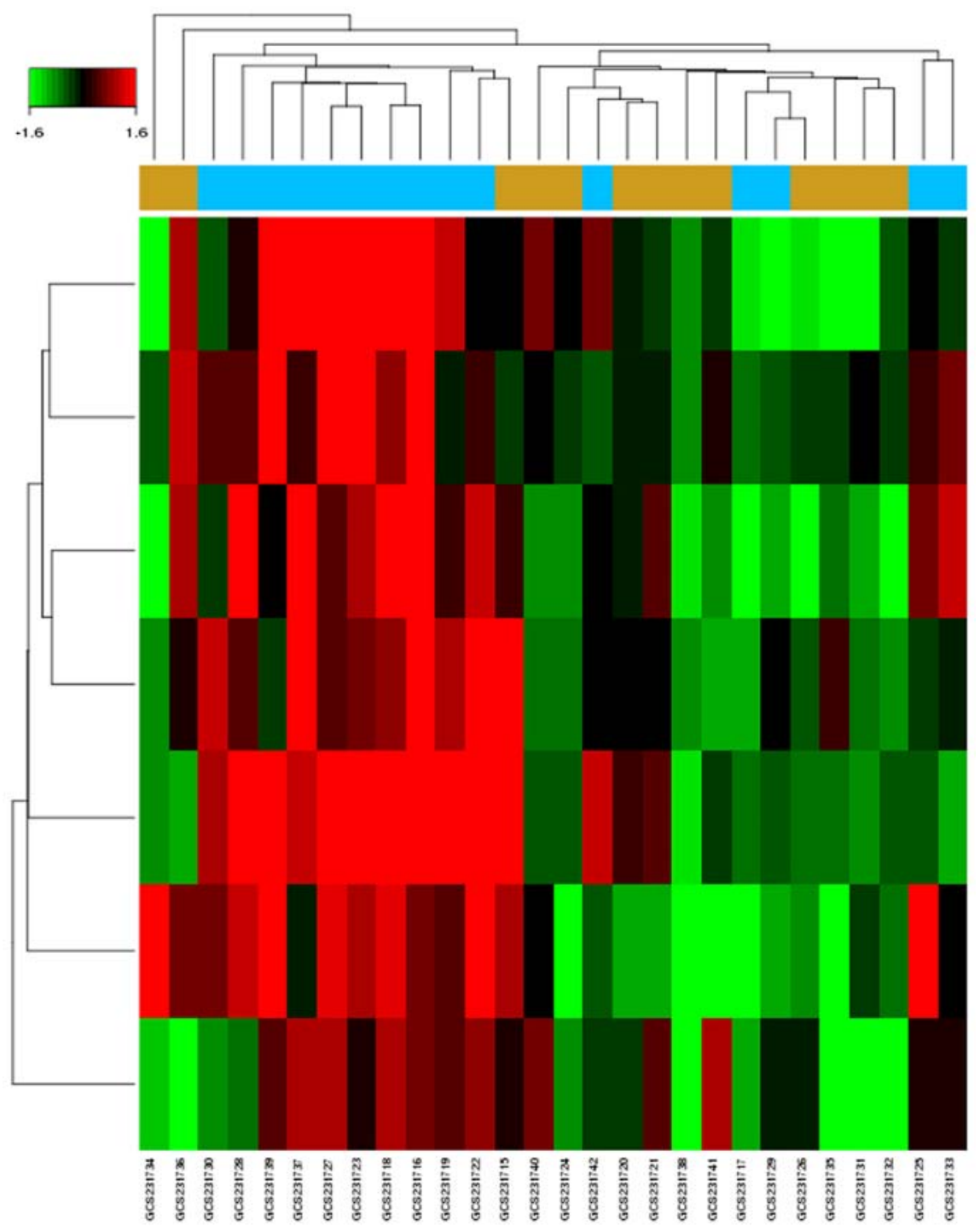

Figure 1. Heat maps of the differentially expressed microRNAs between polycystic ovary syndrome samples and controls from the GSE84376 dataset. Red, upregulated; green, downregulated.

PCOS identified from the microarray dataset (GSE84376; Fig. 1). The dataset contained 15 patients with PCOS and 13 control individuals with male factor infertility. The computational analysis of DE-miRNA target genes based on TargetScan, DIANA-microT-CDS, miRDB and miRTarBase identified 935 genes (Table II). Using a combination of algorithms, this approach provided a more accurate prediction of target genes.

Functional characterization of target genes. GO enrichment analysis was performed for the DE-miRNA target genes using Metascape software. The most significantly enriched gene set was 'homophilic cell adhesion via plasma membrane adhesion molecules' (GO:0007156) (Fig. 2A). The analysis also revealed that PCOS was associated with 'cellular response to nitrogen compound' (GO:1901699), 'blood vessel development' (GO:0001568), 'positive regulation of hydrolase activity' (GO:0051345), 'Axon guidance' (hsa04360), 'regulation of calcium ion transport' (GO:0051924) and 'chloride transmembrane transport' (GO:1902476). Notably, all these biological processes were closely interrelated (Fig. 2B).
PPI network analysis and miRNA-target network. To reduce interference of unrelated genes and to reduce the complexity of the list of PCOS-associated genes, a PPI network was constructed. A total of 32 genes were identified as hub genes with an interaction degree $\geq 10$ (Fig. 3A and Table III). These hub genes were targets of PCOS-associated miRNAs (Fig. 3B). Of these hub genes, mitogen-activated protein kinase 1 (MAPK1) had the highest degree of 46, followed by phosphatase and tensin homolog (PTEN) and cAMP responsive element binding protein 1 (CREB1), with degrees of 36 and 35, respectively. The hub genes identified by the PPI network analysis may serve as potential targets for future research into PCOS treatment. As shown in Fig. 3B, a miRNA-hub gene network was constructed. The hub target genes may be regulated by Homo sapiens (hsa)-miR-3135b, hsa-miR-1225-5p, hsa-miR-1587, hsa-miR-3188, hsa-miR-4433-3p and hsa-miR-4417, with hsa-miR-3135b having the highest correlation degree.

Screening of potential transcription factors. The present study identified the top 10 most significant transcription factors for 
Table II. Differentially expressed miRNAs in polycystic ovary syndrome and their target genes.

\begin{tabular}{|c|c|c|c|}
\hline miRNAs & Fold change [Log(Fold Change $)]$ & P-value & Target gene (number of total target genes) \\
\hline hsa-miR-3188 & 2.781063 & 0.003592 & $\begin{array}{l}\text { HIF1AN ESPL1 SRCAP ZSCAN25 TMBIM6 } \\
\text { TFCP2L1 CSNK2A1 } \\
\text { RPLP1 (131) }\end{array}$ \\
\hline hsa-miR-4433-3p & 2.347565 & 0.003906 & $\begin{array}{l}\text { TNRC6B BZW1 GPR63 DDX52 TMEM59 } \\
\text { STK4 BICD2 TCHHL1 CYB561A3 OLR1 } \\
(205)\end{array}$ \\
\hline hsa-miR-3135b & 2.26603 & 0.006199 & $\begin{array}{lccc}\text { YIPF4 ANKRD36 } & \text { CUBN } & \text { METTL2B } \\
\text { INTS3SIK2 } & \text { FBXO27 } & \text { CCDC142 } & \text { DNAJC10 } \\
\text { ST3GAL1 }(313) & \end{array}$ \\
\hline hsa-miR-1587 & 1.66782 & 0.007895 & $\begin{array}{l}\text { PPM1N SLC2A8 DAO RAD54L2 FBXL16 } \\
\text { CD1B CHRFAM7A GIPC3 PLK2 ZNF609 } \\
(140)\end{array}$ \\
\hline hsa-miR-1225-5p & 1.726874 & 0.008972 & $\begin{array}{llll}\text { PIFO ZNF37A KANSL1L } & \text { KIAA2022 } \\
\text { COL8A1 FAM149B1 ERO1LB } & \text { KIAA0196 } \\
\text { PSME4 ZCCHC12 (55) } & \end{array}$ \\
\hline hsa-miR-4417 & 2.174213 & 0.009638 & $\begin{array}{l}\text { ORAI2 MLF2 CHST2 ATP6V1B1 MTSS1 } \\
\text { SMEK2 STK38 SYCE1 LSM12 KIR2DL4 } \\
(73)\end{array}$ \\
\hline hsa-miR-4749-5p & 2.063519 & 0.009914 & $\begin{array}{llll}\text { NFIX SAP18 } & \text { EMID1 } & \text { GPRC5B } & \text { ZNF558 } \\
\text { FOXRED2 FOXE1 } & \text { ABCE1 } & \text { TMOD3 } \\
\text { FAM84A (18) } & & & \end{array}$ \\
\hline
\end{tabular}

miRNA/miR, microRNA; hsa, homo sapiens.

target genes of miRNAs, based on data from FunRich. These included transcription factor Sp1, myogenic factor 5, homeobox protein $\mathrm{Nkx}-2.1$, nuclear factor I C-type, homeobox protein NOBOX, early growth response protein 1, Ras-responsive element-binding protein 1 , visual system homeobox 2 , T-cell acute lymphocytic leukemia protein 1 and central areolar choroidal dystrophy (Fig. 4).

Patient characteristics. The study population baseline descriptive parameters are summarized in Table I. There were no significant differences in age, infertility duration or menarche age, with the exception of body mass index $(\mathrm{P}=0.002)$. Patients with PCOS exhibited elevated LH levels $(\mathrm{P}=0.005)$ together with significantly low levels of FSH $(\mathrm{P}=0.013)$ in patients with PCOS on the third day of the menstrual cycle. Furthermore, LH/FSH was significantly different $(\mathrm{P}<0.001)$ between the PCOS and control groups. IVF outcomes revealed that the number of retrieved oocytes in the PCOS group was higher than that in the control group $(\mathrm{P}=0.003)$. Furthermore, the fertilization and cleavage rates in the PCOS group were lower than that in control group $(\mathrm{P}<0.05)$.

Validation of candidate miRNAs using RT-qPCR. For confirmation purposes, the seven miRNAs (hsa-miR-3188, hsa-miR-4433-3p, hsa-miR-3135b, hsa-miR-1587, hsa-miR-1225-5p, hsa-miR-4417 and hsa-miR-4749-5p) identified were re-examined using RT-qPCR in the 73 samples (38 patients with PCOS and 35 from the control group) from the validation cohort. As shown in Fig. 5, the levels of hsa-miR-3188 and hsa-miR-3135b were significantly higher in GCs of the PCOS group compared with those of the control group. However, there was no significant difference between the two groups for the levels of hsa-miR-4433-3p, hsa-miR-1587, hsa-miR-1225-5p or hsa-miR-4749-5p. No hsa-miR-4417 expression was detected in GCs (data not shown).

Correlations between validated miRNAs and clinical parameters. Correlation analyses were used to examine the association between miRNAs and clinical parameters of PCOS. The results (data not shown) revealed that the levels of hsa-miR-3135b were positively correlated with the number of oocytes retrieved $(\mathrm{r}=0.450 ; \mathrm{P}<0.001)$ and negatively correlated with fertilization $(\mathrm{r}=-0.280 ; \mathrm{P}=0.016)$ and cleavage rates ( $\mathrm{r}=-0.232 ; \mathrm{P}=0.049)$.

\section{Discussion}

PCOS is an endocrine disorder that affects $6-21 \%$ of reproductive-aged women and $50 \%$ of sub-fertile women (32). PCOS is the leading cause of menstrual complications in women, which involves a polygenic multifactorial model with candidate genes involved in the regulation of the biosynthesis and metabolism of folliculogenesis, androgen, insulin and glucose metabolism (33). However, little is known about its pathogenesis. Examining the molecular mechanism is of great importance for the diagnosis and treatment of PCOS. The identification 
A

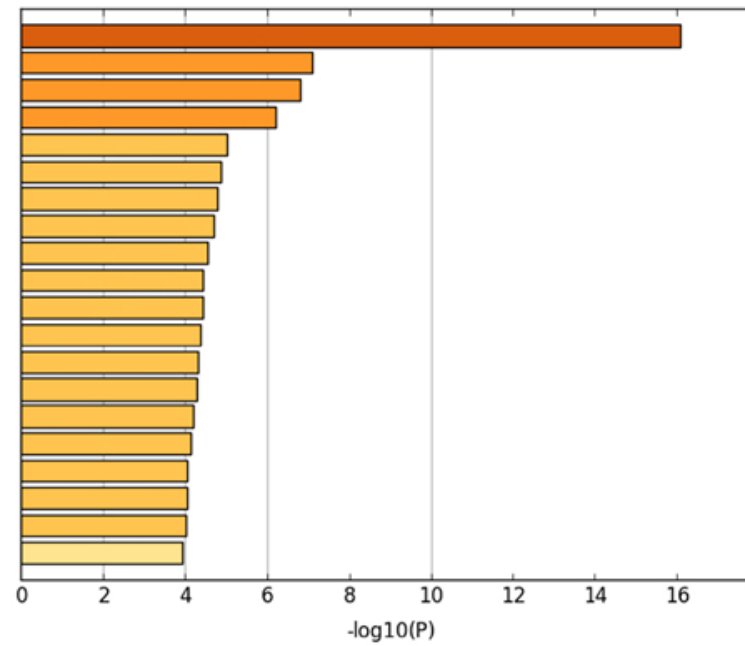

GO:0007156: homophilic cell adhesion via plasma membrane adhesion molecules

GO:1901699: cellular response to nitrogen compound

GO:0061515: myeloid cell development

GO:0031344: regulation of cell projection organization

GO:0001568: blood vessel development

GO:0051345: positive regulation of hydrolase activity

GO:1902475: L-alpha-amino acid transmembrane transport

hsa04360: Axon guidance

GO:0030004: cellular monovalent inorganic cation homeostasis

GO:0051924: regulation of calcium ion transport

GO:0001763: morphogenesis of a branching structure

GO:0030099: myeloid cell differentiation

GO:0007423: sensory organ development

GO:0035264: multicellular organism growth

GO:0060322: head development

GO:0030217: T cell differentiation

GO:0045596: negative regulation of cell differentiation

GO:1902476: chloride transmembrane transport

GO:0007507: heart development

GO:0030324: lung development

B
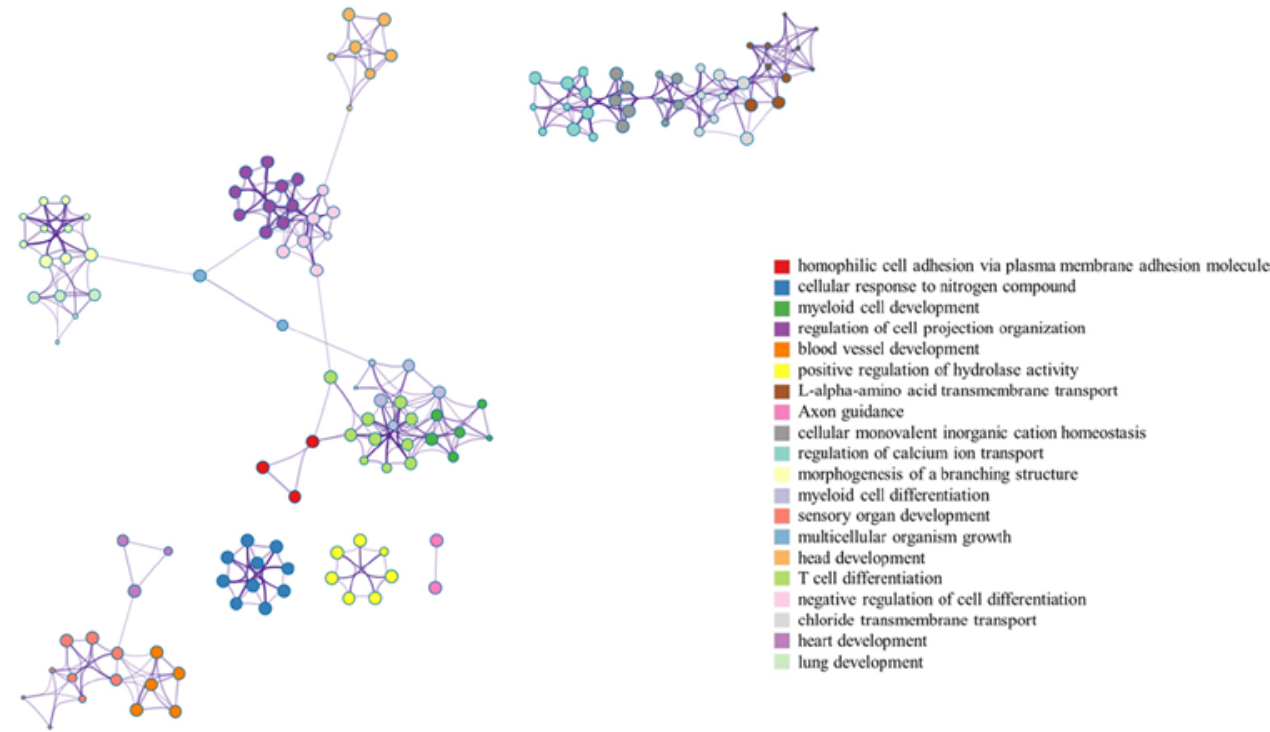

Figure 2. Functional enrichment analysis of miRNA-target genes. (A) Top 20 clusters from Metascape pathway enrichment analysis of differentially expressed-miRNAs-associated genes. (B) Associations between these top 20 clusters enrichment terms displayed as a network analyzed by Metascape. Nodes of the same color belong to the same cluster. Terms with a similarity score $>0.3$ are linked by an edge. The network was visualized with Cytoscape with 'force-directed' layout and edge bundled for clarity. miRNA, microRNA.

of miRNAs involved in PCOS may provide novel insight for detection, diagnosis, treatment and prognosis.

There is accumulating evidence that miRNAs serve important roles in a variety of human diseases, including development, proliferation, cellular differentiation, cell-cycle control and cell death (34). miRNAs participate in the formation of primordial follicles, follicular recruitment and selection, follicular atresia, oocyte-cumulus cell interaction and GC functions (35). Accumulating evidence suggested that miRNAs contribute to the development and progression of PCOS (36). miR-324-3p levels were decreased in a PCOS rat model, while overexpression of miR-324-3p can promote the apoptosis of GCs and inhibit cell proliferation by targeting Wnt family member 2B (37). A previous study reported that a series of miRNAs identified as clinically relevant markers of PCOS are associated with obesity and metabolic dysfunction (38). miR-155 has been used as a biomarker to monitor the efficacy of antiandrogen therapy in a hyperandrogenic patient with PCOS (39). In addition, suppression of miR-19b increased the proliferative ability of GCs by targeting insulin-like growth factor 1 (IGF-1) in PCOS (40). Using Illumina deep sequencing technology, Xue et al (36) identified 263 DE-miRNAs in the follicular fluid of PCOS and control groups, which were involved in the regulation of biological functions and different signaling pathways. In a mouse PCOS model, miR-27a-3p is involved in ovarian follicular development by affecting estradiol and androgen imbalance (41). Previous studies have demonstrated that several miRNAs are regulated by hormones, including LH, hCG and FSH (42-45). These results suggested that miRNAs may participate in the regulation of ovarian functions associated with changes in hormone content. In the present study, based on the GEO dataset (GSE84376), seven DE-miRNAs were identified using bioinformatics analysis.

To date, there are a limited number of studies about the role of miRNAs in patients with PCOS. In the present study, hsa-miR-3188,hsa-miR-4433-3p,hsa-miR-3135b,hsa-miR-1587, hsa-miR-1225-5p, hsa-miR-4417 and hsa-miR-4749-5p were found to be differentially expressed compared with the control group. Zhou et al (46) demonstrated that miR-3188 knockout can inactivate the Notch signaling pathway through 
A

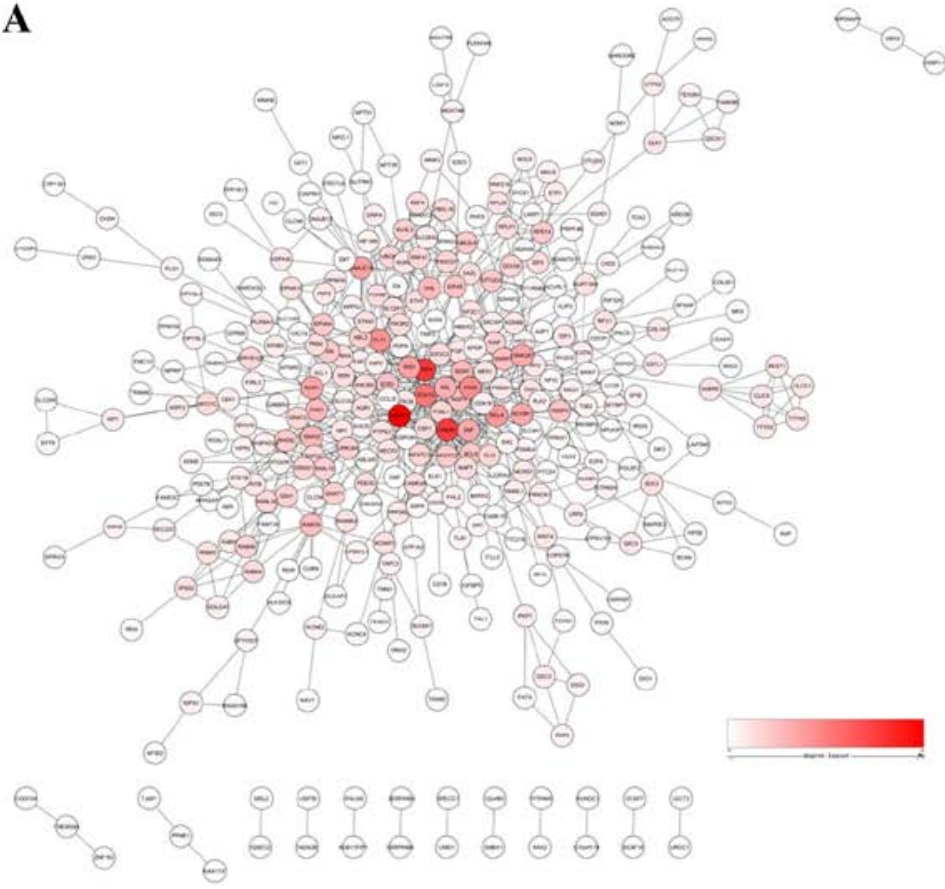

$\mathbf{B}$
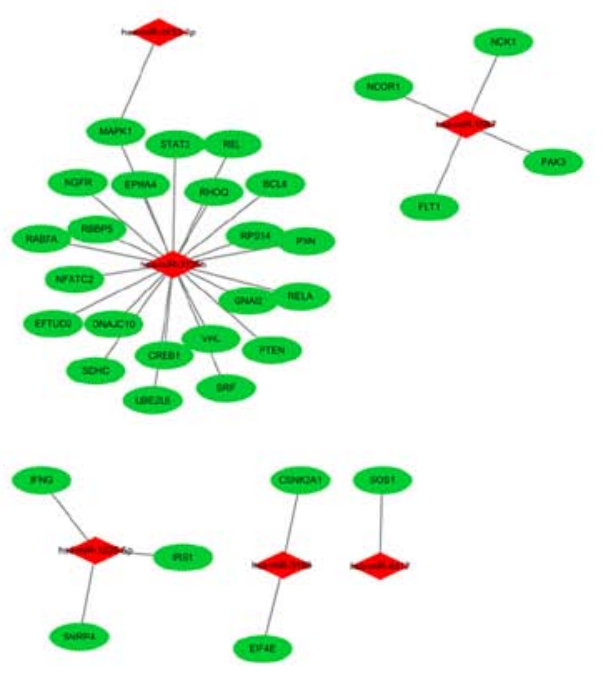

Figure 3. miRNA-gene regulatory network in polycystic ovary syndrome. (A) Protein-protein interaction network of 935 target genes generated using Cytoscape software. (B) miRNA-gene regulatory network based on the hub target genes. Red, miRNAs; green, target genes. miRNA, microRNA.

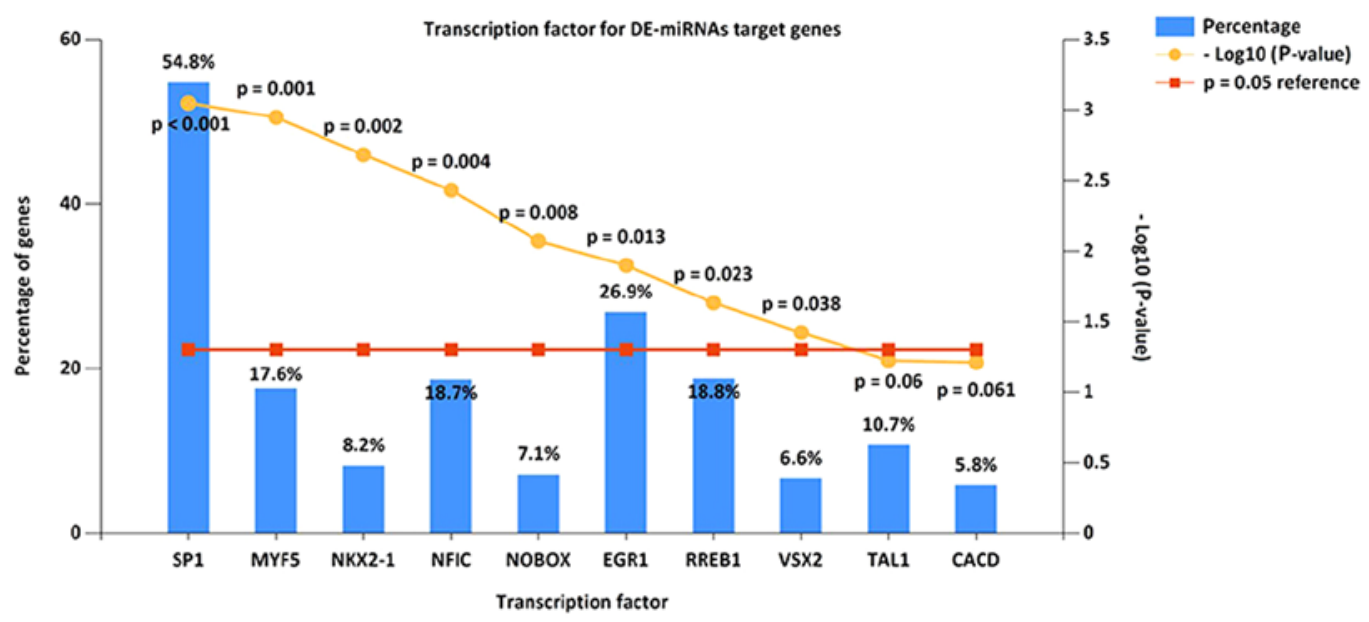

Figure 4. Enriched transcription factors by differentially expressed microRNAs target genes. The top 10 most significant transcription factors include Sp1, MYF5, NKX2-1, NFIC, NOBOX, EGR1, RREB1, VSX2, TAL1 and CACD. Sp1, transcription factor Sp1; MYF5, myogenic factor 5; NKX2-1, homeobox protein Nkx-2.1; NFIC, nuclear factor I C-type; NOBOX, homeobox protein NOBOX; EGR1, early growth response protein 1; RREB1, Ras-responsive element-binding protein 1; VSX2, visual system homeobox 2; TAL1, T-cell acute lymphocytic leukemia protein 1; CACD, central areolar choroidal dystrophy.

upregulation of Zinc-fingers and homeoboxes 2 in vivo and in vitro, thus inhibiting the growth and metastasis of hepatocellular carcinoma cells. By contrast, upregulation of miR-3188 regulates cancer cell proliferation, apoptosis and migration in breast cancer by targeting tumor-suppressor candidate 5 and activating the p38 MAPK signaling pathway (47). In a previous study on nasopharyngeal carcinoma, miR-3188 regulated cell proliferation and chemosensitivity through a forkhead box $\mathrm{O} 1$ (FOXO1)-modulated positive feedback loop with mammalian target of rapamycin-p-PI3K/AKT-c-JUN (48). Liu et al (49) reported a high expression of miR-3135b in patients with coronary calcification. Figueroa et al (50) identified miR-1587 as a mediator of exosomes that can regulate tumor-initiating glioma stem-like cells by suppressing nuclear receptor corepressor 1. In human laryngeal carcinoma tissues, Sun et al (51) reported that miR-1225-5p promoted a $\mathrm{G}_{1} / \mathrm{S}$ cell cycle arrest and enhanced cell death. In addition, a previous study revealed that OTU deubiquitinase 5 suppression via hsa-miR-4417 and hsa-miR-6782 could maintain the viability of tumor cells and suppress programmed cell death (52). Song et al (53) demonstrated that, in hepatocellular carcinoma cells, miR-4417 can promote proliferation and suppress apoptosis by targeting tripartite motif-containing 35 and regulating the phosphorylation of pyruvate kinase muscle. Furthermore, the present study 
Table III. Hub genes identified in the protein interaction network.

\begin{tabular}{|c|c|c|c|}
\hline Name & Degree & Betweenness & Closeness \\
\hline MAPK1 & 46 & 0.23654846 & 0.39900249 \\
\hline PTEN & 36 & 0.22614707 & 0.39653036 \\
\hline CREB1 & 35 & 0.18022213 & 0.3874092 \\
\hline STAT3 & 25 & 0.05874048 & 0.3567447 \\
\hline IFNG & 21 & 0.07357852 & 0.33264033 \\
\hline FLT1 & 20 & 0.06162387 & 0.35087719 \\
\hline RELA & 19 & 0.03995804 & 0.34042553 \\
\hline IRS1 & 17 & 0.02215209 & 0.34820457 \\
\hline DNAJC10 & 17 & 0.06587252 & 0.29767442 \\
\hline CSNK2A1 & 16 & 0.07117248 & 0.34408602 \\
\hline NCOR1 & 16 & 0.05439941 & 0.31496063 \\
\hline NCK1 & 16 & 0.04186164 & 0.31714569 \\
\hline SRF & 15 & 0.01578066 & 0.33229491 \\
\hline REL & 13 & 0.01440785 & 0.34042553 \\
\hline PAK3 & 13 & 0.02088241 & 0.31037827 \\
\hline VHL & 13 & 0.02583072 & 0.30798845 \\
\hline GNAI2 & 13 & 0.0176689 & 0.30563515 \\
\hline SDHC & 13 & 0.03731446 & 0.33970276 \\
\hline SNRPA & 12 & 0.07384247 & 0.33826638 \\
\hline SOS1 & 12 & 0.02049813 & 0.33862434 \\
\hline RAB7A & 12 & 0.07246042 & 0.30828516 \\
\hline RBBP5 & 11 & 0.01080627 & 0.2640264 \\
\hline EIF4E & 11 & 0.06378144 & 0.33229491 \\
\hline NGFR & 11 & 0.02044841 & 0.32753327 \\
\hline BCL6 & 11 & 0.0410574 & 0.34115139 \\
\hline RPS14 & 10 & 0.04376657 & 0.27633851 \\
\hline EPHA4 & 10 & 0.01262707 & 0.30710173 \\
\hline PXN & 10 & 0.01479177 & 0.32520325 \\
\hline EFTUD2 & 10 & 0.02160464 & 0.28725314 \\
\hline UBE2L6 & 10 & 0.00749018 & 0.26380874 \\
\hline NFATC2 & 10 & 0.00103466 & 0.31840796 \\
\hline RHOQ & 10 & 0.02818871 & 0.28193833 \\
\hline
\end{tabular}

identified 935 target genes using four databases. To better understand the functions of miRNA-target genes, the present study performed GO analysis.

The GO enrichment analysis revealed that the most significantly enriched gene set was 'homophilic cell adhesion via plasma membrane adhesion molecules'. The protocadherins (PCDHs), the largest group of the cadherin superfamily, were identified as the main target genes of the DE-miRNAs and were divided into two major groups: 'Clustered' PCDHs that consisted of PCDH- $\alpha$, PCDH- $\beta$ and PCDH- $\gamma$, and 'non-clustered' PCDHs (54). Previous studies have reported that PCDHs are important for embryonic and neural development, and for the etiology and progression of multiple types of cancer $(55,56)$. Inan et al (57) analyzed the whole gene expression of GCs from a patient with recurrent empty follicle syndrome (EFS) using an Affymetrix GeneChip ${ }^{\circledR}$ and observed that PCDH17 was differentially expressed in EFS compared with the control.

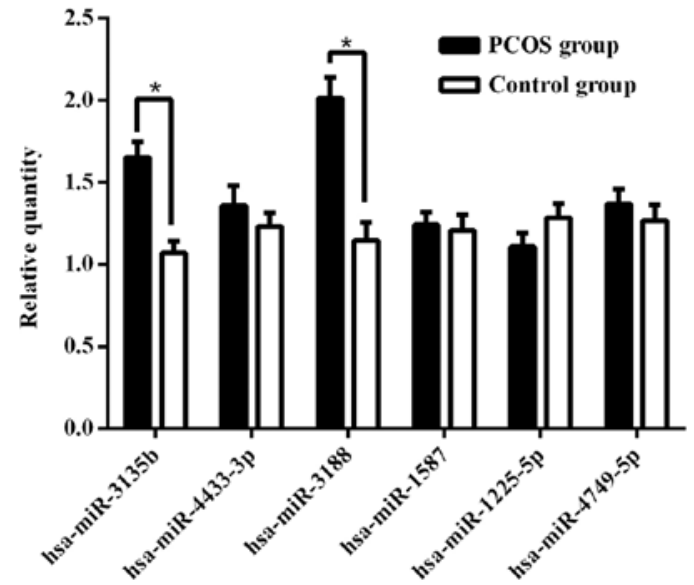

Figure 5. Comparison of the relative expression levels of differentially expressed miRNAs between the polycystic ovary syndrome and control groups. miRNAs verified by reverse transcription-quantitative $\mathrm{PCR}$. ${ }^{*} \mathrm{P}<0.05$. miRNA, microRNA.

Dickinson et al (58) analyzed the effect of pre-ovulatory follicle size on oocyte transcript abundance in beef cows. A total of 106 transcripts were determined to be differentially abundant, of which PCDH $\gamma$ subfamily A 11 was in a higher abundance in small follicle oocyte pools. In addition, the present study identified a series of biological processes associated with PCOS, including 'cellular response to nitrogen compound', 'blood vessel development' and 'regulation of calcium ion and chloride transmembrane transport'. Previous studies have revealed that follicular development disorder, ovulation inhibition and $\mathrm{GC}$ apoptosis are associated with the downregulation of nitric oxide $(59,60)$. Liu et al $(61)$ indicated that the abnormal expression patterns of angiopoietin-like (ANGPT) 1 and ANGPTL2 mRNAs in cumulus cells are associated with impaired oocyte developmental competence in PCOS, via pathological angiogenesis and metabolism. Chen et al (62) demonstrated that impaired cystic fibrosis transmembrane conductance regulator (a chloride ion channel) resulted in ovarian disorders by amplifying FSH-stimulated estrogen production in cystic fibrosis and PCOS. In addition, transmembrane member 16A, a calcium-activated chloride channel, regulates the morphology of oocytes and affects the synthesis of estrogen through the MAPK kinase/ERK signaling cascade (63). These findings provide novel insights into the molecular mechanisms of the regulation of folliculogenesis and ovulation in PCOS.

Furthermore, a PPI network was constructed in the present study, and MAPK1 was identified as the hub gene with the highest connectivity degree $(=46)$, followed by PTEN $(=36)$ and CREB1 (=35). In 2017, Li et al (64) reported that MAPK1, epidermal growth factor receptor, receptor tyrosine-protein kinase ERBB2, FOXO1, NF-к $\beta 1$, IGF-1, cellular tumor antigen P53 and MAPK9 may activate autophagy in the ovarian tissue of patients with PCOS. Cui et al (65) indicated that the MAPK signaling pathway promoted androgen receptor (AR) nuclear translocation, thus influencing AR activity. In a PCOS-prone metabolic syndrome rodent model, metformin appeared to decrease fasting plasma glucose, insulin and homeostatic model assessment-insulin resistance via the MAPK1, AKT2 and 5'-AMP-activated protein kinase catalytic subunit $\alpha 2$ signaling pathways (66). These data supported the hypothesis 
that MAPK1 may be a candidate target gene associated with PCOS. Based on the hub genes identified, the miRNA-gene regulatory network in the present study revealed that hsa-miR-3135b had the highest correlation with the hub genes. In summary, hsa-miR-3135b may participate in the development of PCOS by regulating the expression of hub genes.

In order to better understand the mechanisms of target genes in DE-miRNAs, the present study screened possible transcription factors. Specificity Protein 1 (SP1) is the most common transcription factor, a recent study indicated that SP1 serves an important role in regulating vascular endothelial growth factor (VEGF) production by binding to specific sites in the VEGF promoter (67). Previous studies suggested that SP1 may be involved in the mechanism of insulin resistance in patients with PCOS by mediating the gene expression of insulin $(68,69)$. Anjali et al (69) revealed that FSH can stimulate the expression of insulin receptor substrate 2 in human GCs through cAMP/SP1 in patients with PCOS. Enrichment of SP1 by the target genes of DE-miRNAs suggests their potential involvement in the development and progression of PCOS.

Based on the findings of this present study, only hsa-miR-3188 and hsa-miR-3135b were found to display significantly high levels in GCs of patients with PCOS compared with controls with male factor infertility. An association was found between hsa-miR-3135b and the hub genes, the expression level of hsa-miR-3135b was observed to be significantly correlated with the number of oocytes retrieved, fertilization and cleavage rate $(\mathrm{P}<0.05)$. Overall, these results demonstrate that hsa-miR-3135b may participate in the development and maturation of oocytes by the regulation of target genes.

There are several limitations to the present study. First, the study is based on microarray data from GSE84376. The sample size is small; thus, further studies are necessary to investigate additional samples from multiple centers. Second, various enriched functions and hub genes were identified in the present study; however, their associations were not fully elucidated. In conclusion, the data presented provide a comprehensive analysis of DE-miRNAs that may be involved in the development of PCOS. The DE-miRNAs and hub target genes identified in the present study may be used in the future as targets for PCOS prevention and treatment. However, their biological functions and mechanism of action in PCOS require further investigation.

\section{Acknowledgements}

Not applicable.

\section{Funding}

The present study was supported by the project sponsored by the Research Fund of National Key Research and Development Program (grant no. 2018YFC1002103) and the Research Fund of National Health and Family Planning Commission of China (grant no. RFNHFPCC, 201402004).

\section{Availability of data and materials}

The dataset used and/or analyzed in the present study is available from the corresponding author on reasonable request.

\section{Authors' contributions}

XW conceived and supervised the project. YH and SX performed the experiments. YW and GQ analyzed the data. YH, YW and XW wrote the manuscript. All the authors reviewed the manuscript.

\section{Ethics approval and consent to participate}

The study was approved by the Ethics Committee of Children's Hospital of Shanxi and Women Health Center of Shanxi. Written informed consent for inclusion was obtained from each participant.

\section{Patient consent for publication}

Not applicable.

\section{Competing interests}

The authors declare that they have no competing interests.

\section{References}

1. Franks S: Polycystic ovary syndrome. N Engl J Med 333: 853-861, 1995.

2. Lizneva D, Suturina L, Walker W, Brakta S, Gavrilova-Jordan L and Azziz R: Criteria, prevalence, and phenotypes of polycystic ovary syndrome. Fertil Steril 106: 6-15, 2016.

3. Zhao Y and Qiao J: Ethnic differences in the phenotypic expression of polycystic ovary syndrome. Steroids 78: 755-760, 2013.

4. Dumesic DA, Padmanabhan V and Abbott DH: Polycystic ovary syndrome and oocyte developmental competence. Obstet Gynecol Surv 63: 39-48, 2008.

5. Nelson LR and Bulun SE: Estrogen production and action. J Am Acad Dermatol 45 (3 Suppl): S116-S124, 2001.

6. Artimani T, Saidijam M, Aflatoonian R, Amiri I, Ashrafi M, Shabab N, Mohammadpour N and Mehdizadeh M: Estrogen and progesterone receptor subtype expression in granulosa cells from women with polycystic ovary syndrome. Gynecol Endocrinol 31: 379-383, 2015.

7. Haouzi D, Assou S, Monzo C, Vincens C, Dechaud H and Hamamah S: Altered gene expression profile in cumulus cells of mature MII oocytes from patients with polycystic ovary syndrome. Hum Reprod 27: 3523-3530, 2012.

8. Jansen E, Laven JS, Dommerholt HB, Polman J, van Rijt C, van den Hurk C, Westland J, Mosselman S and Fauser BC: Abnormal gene expression profiles in human ovaries from polycystic ovary syndrome patients. Mol Endocrinol 18: 3050-3063, 2004.

9. Manneras-Holm L, Benrick A and Stener-Victorin E: Gene expression in subcutaneous adipose tissue differs in women with polycystic ovary syndrome and controls matched pair-wise for age, body weight, and body mass index. Adipocyte 3: 190-196, 2014.

10. Sorensen AE, Wissing ML, Salö S, Englund AL and Dalgaard LT: MicroRNAs related to polycystic ovary syndrome (PCOS). Genes (Basel) 5: 684-708, 2014.

11. Wood JR, Ho CK, Nelson-Degrave VL, McAllister JM and Strauss JF III: The molecular signature of polycystic ovary syndrome (PCOS) theca cells defined by gene expression profiling. J Reprod Immunol 63: 51-60, 2004.

12. Esteller M: Non-coding RNAs in human disease. Nat Rev Genet 12: 861-874, 2011

13. Rossi JJ: New hope for a microRNA therapy for liver cancer. Cell 137: 990-992, 2009.

14. Kim YJ, Ku SY, Kim YY, Liu HC, Chi SW, Kim SH, Choi YM, Kim JG and Moon SY: MicroRNAs transfected into granulosa cells may regulate oocyte meiotic competence during in vitro maturation of mouse follicles. Hum Reprod 28: 3050-3061, 2013.

15. Xu B, Zhang YW, Tong XH and Liu YS: Characterization of microRNA profile in human cumulus granulosa cells: Identification of microRNAs that regulate Notch signaling and are associated with PCOS. Mol Cell Endocrinol 404: 26-36, 2015. 
16. Imbar $\mathrm{T}$ and Eisenberg I: Regulatory role of microRNAs in ovarian function. Fertil Steril 101: 1524-1530, 2014.

17. Liu J, Tu F, Yao W, Li X, Xie Z, Liu H, Li Q and Pan Z: Conserved miR-26b enhances ovarian granulosa cell apoptosis through HAS2-HA-CD44-Caspase-3 pathway by targeting HAS2. Sci Rep 6: 21197, 2016.

18. Zhou J, Liu J, Pan Z, Du X, Li X, Ma B, Yao W, Li Q and Liu H: The let-7g microRNA promotes follicular granulosa cell apoptosis by targeting transforming growth factor-beta type 1 receptor. Mol Cell Endocrinol 409: 103-112, 2015.

19. Carletti MZ and Christenson LK: MicroRNA in the ovary and female reproductive tract. J Anim Sci 87 (14 Suppl): E29-E38, 2009

20. Chen YH, Heneidi S, Lee JM, Layman LC, Stepp DW, Gamboa GM, Chen BS, Chazenbalk G and Azziz R: miRNA-93 inhibits GLUT4 and is overexpressed in adipose tissue of polycystic ovary syndrome patients and women with insulin resistance. Diabetes 62: 2278-2286, 2013.

21. Long W, Zhao C, Ji C, Ding H, Cui Y, Guo X, Shen R and Liu J: Characterization of serum microRNAs profile of PCOS and identification of novel non-invasive biomarkers. Cell Physiol Biochem 33: 1304-1315, 2014.

22. Roth LW, McCallie B, Alvero R, Schoolcraft WB, Minjarez D and Katz-Jaffe MG: Altered microRNA and gene expression in the follicular fluid of women with polycystic ovary syndrome. J Assist Reprod Genet 31: 355-362, 2014.

23. Murri M, Insenser M, Fernandez-Duran E, San-Millan JL and Escobar-Morreale HF: Effects of polycystic ovary syndrome (PCOS), sex hormones, and obesity on circulating miRNA-21, miRNA-27b, miRNA-103, and miRNA-155 expression. J Clin Endocrinol Metab 98: E1835-E1844, 2013.

24. Shi L, Liu S, Zhao W and Shi J: miR-483-5p and miR-486-5p are down-regulated in cumulus cells of metaphase II oocytes from women with polycystic ovary syndrome. Reprod Biomed Online 31: 565-572, 2015.

25. Jiang L, Huang J, Li L, Chen Y, Chen X, Zhao X and Yang D: MicroRNA-93 promotes ovarian granulosa cells proliferation through targeting CDKN1A in polycystic ovarian syndrome. J Clin Endocrinol Metab 100: E729-E738, 2015.

26. Zhu XL, Chen XF and Xu WL: miR-34a regulates apoptosis of human ovarian granulosa cells. J Jiangsu Univ (Med Ed) 26: 470-474, 2016 (In Chinese).

27. He $\mathrm{X}$ and Zhang J: Why do hubs tend to be essential in protein networks? PLoS Genet 2: e88, 2006.

28. Franceschini A, Szklarczyk D, Frankild S, Kuhn M, Simonovic M, Roth A, Lin J, Minguez P, Bork P, von Mering C and Jensen LJ STRING v9.1: Protein-protein interaction networks, with increased coverage and integration. Nucleic Acids Res 41 (Database Issue): D808-D815, 2013.

29. Pathan M, Keerthikumar S, Ang CS, Gangoda L, Quek CY, Williamson NA, Mouradov D, Sieber OM, Simpson RJ, Salim A, et al: FunRich: An open access standalone functional enrichment and interaction network analysis tool. Proteomics 15 : 2597-2601, 2015.

30. Rotterdam ESHRE/ASRM-Sponsored PCOS consensus workshop group: Revised 2003 consensus on diagnostic criteria and long-term health risks related to polycystic ovary syndrome (PCOS). Hum Reprod 19: 41-47, 2004.

31. Livak KJ and Schmittgen TD: Analysis of relative gene expression data using real-time quantitative PCR and the 2(-Delta Delta C(T)) method. Methods 25: 402-408, 2001.

32. Azziz R, Carmina E, Dewailly D, Diamanti-Kandarakis E, Escobar-Morreale HF, Futterweit W, Janssen OE, Legro RS Norman RJ, Taylor AE, et al: Positions statement: Criteria for defining polycystic ovary syndrome as a predominantly hyperandrogenic syndrome: An Androgen Excess Society guideline. J Clin Endocrinol Metab 91: 4237-4245, 2006.

33. Escobar-Morreale HF, Luque-Ramirez M and San Millan JL: The molecular-genetic basis of functional hyperandrogenism and the polycystic ovary syndrome. Endocr Rev 26: 251-282, 2005.

34. Miska EA: How microRNAs control cell division, differentiation and death. Curr Opin Genet Dev 15: 563-568, 2005.

35. Maalouf SW, Liu WS and Pate JL: MicroRNA in ovarian function. Cell Tissue Res 363: 7-18, 2016.

36. Xue Y,Lv J, Xu P, Gu L, Cao J, Xu L, Xue K and Li Q: Identification of microRNAs and genes associated with hyperandrogenism in the follicular fluid of women with polycystic ovary syndrome. J Cell Biochem 119: 3913-3921, 2018.

37. Jiang YC and Ma JX: The role of MiR-324-3p in polycystic ovary syndrome (PCOS) via targeting WNT2B. Eur Rev Med Pharmacol Sci 22: 3286-3293, 2018
38. Murri M, Insenser M, Fernandez-Duran E, San-Millan JL, Luque-Ramirez M and Escobar-Morreale HF: Non-targeted profiling of circulating microRNAs in women with polycystic ovary syndrome (PCOS): Effects of obesity and sex hormones. Metabolism 86: 49-60, 2018.

39. Arancio W, Calogero Amato M, Magliozzo M, Pizzolanti G, Vesco R and Giordano C: Serum miRNAs in women affected by hyperandrogenic polycystic ovary syndrome: The potential role of miR-155 as a biomarker for monitoring the estroprogestinic treatment. Gynecol Endocrinol 34: 704-708, 2018.

40. Zhong Z, Li F, Li Y, Qin S, Wen C, Fu Y and Xiao Q: Inhibition of microRNA-19b promotes ovarian granulosa cell proliferation by targeting IGF-1 in polycystic ovary syndrome. Mol Med Rep 17: 4889-4898, 2018.

41. Wang M, Liu M, Sun J, Jia L, Ma S, Gao J, Xu Y, Zhang H, Tsang SY and Li X: MicroRNA-27a-3p affects estradiol and androgen imbalance by targeting Crebl in the granulosa cells in mouse polycytic ovary syndrome model. Reprod Biol 17: 295-304, 2017.

42. YaoG,YinM,Lian J,Tian H,LiuL,LiX and Sun F: MicroRNA-224 is involved in transforming growth factor-beta-mediated mouse granulosa cell proliferation and granulosa cell function by targeting Smad4. Mol Endocrinol 24: 540-551, 2010.

43. Carletti MZ, Fiedler SD and Christenson LK: MicroRNA 21 blocks apoptosis in mouse periovulatory granulosa cells. Biol Reprod 83: 286-295, 2010.

44. Yin M, Wang X, Yao G, Lü M, Liang M, Sun Y and Sun F: Transactivation of micrornA-320 by microRNA-383 regulates granulosa cell functions by targeting E2F1 and SF-1 proteins. J Biol Chem 289: 18239-18257, 2014.

45. Fiedler SD, Carletti MZ, Hong X and Christenson LK: Hormonal regulation of MicroRNA expression in periovulatory mouse mural granulosa cells. Biol Reprod 79: 1030-1037, 2008.

46. Zhou SJ, Deng YL, Liang HF, Jaoude JC and Liu FY: Hepatitis $B$ virus $X$ protein promotes CREB-mediated activation of miR-3188 and Notch signaling in hepatocellular carcinoma. Cell Death Differ 24: 1577-1587, 2017.

47. Chen $X$ and Chen J: MiR-3188 regulates cell proliferation, apoptosis, and migration in breast cancer by targeting TUSC5 and regulating the p38 MAPK signaling pathway. Oncol Res 26: 363-372, 2018 .

48. Zhao M, Luo R, Liu Y, Gao L, Fu Z, Fu Q, Luo X, Chen Y, Deng X, Liang Z, et al: miR-3188 regulates nasopharyngeal carcinoma proliferation and chemosensitivity through a FOXO1-modulated positive feedback loop with mTOR-p-PI3K/AKT-c-JUN. Nat Commun 7: 11309, 2016.

49. Liu W, Ling S, Sun W, Liu T, Li Y, Zhong G, Zhao D, Zhang P, Song J, Jin X, et al: Circulating microRNAs correlated with the level of coronary artery calcification in symptomatic patients. Sci Rep 5: 16099, 2015.

50. Figueroa J, Phillips LM, Shahar T, Hossain A, Gumin J, Kim H, Bean AJ, Calin GA, Fueyo J, Walters ET, et al: Exosomes from Glioma-associated mesenchymal stem cells increase the tumorigenicity of glioma stem-like cells via transfer of miR-1587. Cancer Res 77: 5808-5819, 2017.

51. Sun P, Zhang D, Huang H, Yu Y, Yang Z, Niu Y and Liu J: MicroRNA-1225-5p acts as a tumor-suppressor in laryngeal cancer via targeting CDC14B. Biol Chem 400: 237-246, 2019.

52. Fomicheva KA, Osip'yants AI, Knyazev EN, Samatov TR and Shkurnikov MY: Detection of potential metastatic prostate cancer circulating biomarkers by comparison of miRNA profiles in DU145 cells and culture medium. Bull Exp Biol Med 162: 792-796, 2017.

53. Song L, Zhang W, Chang Z, Pan Y, Zong H, Fan Q and Wang L miR-4417 targets tripartite motif-containing 35 (TRIM35) and regulates pyruvate kinase muscle 2 (PKM2) phosphorylation to promote proliferation and suppress apoptosis in hepatocellular carcinoma cells. Med Sci Monit 23: 1741-1750, 2017.

54. Schreiner D and Weiner JA: Combinatorial homophilic interaction between gamma-protocadherin multimers greatly expands the molecular diversity of cell adhesion. Proc Natl Acad Sci USA 107: 14893-14898, 2010.

55. Yagi T and Takeichi M: Cadherin superfamily genes: Functions, genomic organization, and neurologic diversity. Genes Dev 14: $1169-1180,2000$.

56. Mah KM and Weiner JA: Regulation of Wnt signaling by protocadherins. Semin Cell Dev Biol 69: 158-171, 2017.

57. Inan MS, Al-Hassan S, Ozand P and Coskun S: Transcriptional profiling of granulosa cells from a patient with recurrent empty follicle syndrome. Reprod Biomed Online 13: 481-491, 2006. 
58. Dickinson SE: Effect of pre-ovulatory follicle size on oocyte transcript abundance in beef cows. Master's Thesis University of Missouri-Columbia, 2016.

59. Mitchell LM, Kennedy CR and Hartshorne GM: Expression of nitric oxide synthase and effect of substrate manipulation of the nitric oxide pathway in mouse ovarian follicles. Hum Reprod 19: 30-40, 2004

60. Guo YX, Zhang GM, Yao XL, Tong R, Cheng CY, Zhang TT, Wang ST, Yang H and Wang F: Effects of nitric oxide on steroidogenesis and apoptosis in goat luteinized granulosa cells. Theriogenology 126: 55-62, 2018.

61. Liu Z, Liu C, Hao C, Xue Q, Huang X, Zhang N, Bao H and Qu Q: Aberrant expression of angiopoietin-like proteins 1 and 2 in cumulus cells is potentially associated with impaired oocyte developmental competence in polycystic ovary syndrome. Gynecol Endocrinol 32: 557-561, 2016.

62. Chen H, Guo JH, Lu YC, Ding GL, Yu MK, Tsang LL, Fok KL, Liu XM,Zhang XH, Chung YW, et al: Impaired CFTR-dependent amplification of FSH-stimulated estrogen production in cystic fibrosis and PCOS. J Clin Endocrinol Metab 97: 923-932, 2012.

63. Sun M, Sui Y, Li L, Su W, Hao F, Zhu Q, Di W, Gao H and Ma T: Anoctamin 1 calcium-activated chloride channel downregulates estrogen production in mouse ovarian granulosa cells. Endocrinology 155: 2787-2796, 2014.

64. Li D, You Y, Bi FF, Zhang TN, Jiao J, Wang TR, Zhou YM, Shen ZQ, Wang XX and Yang Q: Autophagy is activated in the ovarian tissue of polycystic ovary syndrome. Reproduction 155: $85-92,2018$

65. Cui Y, Sun Y, Hu S, Luo J, Li L, Li X, Yeh S, Jin J and Chang C: Neuroendocrine prostate cancer (NEPCa) increased the neighboring $\mathrm{PCa}$ chemoresistance via altering the $\mathrm{PTHrP} / \mathrm{p} 38 /$ Hsp27/androgen receptor (AR)/p21 signals. Oncogene 35: 6065-6076, 2016.
66. Kupreeva M, Diane A, Lehner R, Watts R, Ghosh M, Proctor S and Vine D: Effect of metformin and flutamide on insulin, lipogenic and androgen-estrogen signaling and cardiometabolic risk in a PCOS-prone rodent model. Am J Physiol Endocrinol Metab 316: E16-E33, 2019.

67. Liu N, Ding D, Hao W, Yang F, Wu X, Wang M, Xu X, Ju Z, Liu JP, Song Z, et al: hTERT promotes tumor angiogenesis by activating VEGF via interactions with the Sp1 transcription factor. Nucleic Acids Res 44: 8693-8703, 2016.

68. Solomon SS, Majumdar G, Martinez-Hernandez A and Raghow R: A critical role of Sp1 transcription factor in regulating gene expression in response to insulin and other hormones. Life Sci 83: 305-312, 2008

69. Anjali G, Kaur S, Lakra R, Taneja J, Kalsey GS, Nagendra A, Shrivastav TG, Devi MG, Malhotra N, Kriplani A and Singh R: FSH stimulates IRS-2 expression in human granulosa cells through cAMP/SP1, an inoperative FSH action in PCOS patients. Cell Signal 27: 2452-2466, 2015.

(i) $($ This work is licensed under a Creative Common Attribution-NonCommercial-NoDerivatives 4.0 International (CC BY-NC-ND 4.0) License. 